\title{
Deep Spectroscopy in the Field of 3C 212
}

\author{
Alan Stockton \\ Institute for Astronomy, University of Hawaii, 2680 Woodlawn Drive, Honolulu, HI 96822 \\ Susan E. Ridgway \\ Department of Physics, University of Oxford, Nuclear and Astrophysics Laboratory, Keble Road, \\ Oxford, OX13RH, UK
}

\begin{abstract}
We present the results of longslit and multiaperture spectroscopy of faint galaxies in the field of the $z=1.049$ quasar $3 \mathrm{C} 212$. We show that an apparently aligned optical feature beyond the NW radio lobe has a redshift $z=0.928$, and a similarly aligned feature just beyond the SE radio lobe has a redshift $z=1.053$, quite close to that of the quasar. While the NW optical component is extremely well aligned with the radio jet and has a morphology that is very similar to that of the radio lobe lying $3^{\prime \prime}$ interior to it, the fact that we find three other field galaxies with closely similar redshifts indicates that it is most likely a member of an intervening group rather than an unusual example of true alignment with the radio structure. In addition, we have found two galaxies (besides the one near the SE radio lobe) having redshifts close to that of $3 \mathrm{C} 212$. We have firm or probable redshifts for 66 out of 82 galaxies we have observed in this field. Comparison with the redshift distribution of faint field galaxies indicates that a large fraction of the remaining 16 galaxies are likely to be at redshifts $\gtrsim$ 1.4. There are at least two low-redshift dwarf starburst galaxies showing low metal abundance and high ionization in our sample; such galaxies are likely to be relatively common in very faint samples, and, under certain conditions, they could be confused with high-redshift objects.
\end{abstract}

\section{Introduction}

The alignment of the extended optical continuum and emission-line radiation with the radio axis in powerful radio galaxies at high redshifts (Chambers, Miley, \& van Breugel 1987; McCarthy et al. 1987) continues to be a vexing issue in our understanding of the nature and evolution of such objects. In spite of strong evidence that a significant portion of this alignment effect in some objects stems from scattering of radiation from a hidden quasar nucleus into our line of sight (di Serego Aligheri, Cimatti, \& Fosbury 1994; Dickinson, Dey, \& Spinrad 1996), there is also evidence that such scattering is by no means the whole story (Longair, Best, \& Röttgering 1995; Stockton, Ridgway, \& Kellogg 1996). We report elsewhere (Ridgway \& Stockton 1997; hereafter Paper 1) the results of an HST and ground-based imaging survey of a complete sample of $z \sim 1$ 3CR quasars 
and radio galaxies, including some of the first clear examples of the alignment effect seen in quasars (in fact, all 5 of the quasars in our sample show some form of alignment between the optical and radio structure). However, instead of clarifying the nature of the alignment effect, these quasars actually complicate the picture somewhat, in that at least 3 of them show examples of aligned optical structure that has such close and detailed correspondence with the radio structure that it almost certainly is due to optical synchrotron radiation. We do not see similar types of alignment in our radio-galaxy sample, presumably because the aligned synchrotron emission is strongly beamed and the radio jets in the quasars are closer to our line of sight.

One of the quasars showing this type of alignment is $3 \mathrm{C} 212$. Figure $11 A$ shows our HST WFPC2 image with 3.6-cm VLA contours superposed (see also Fig. 6 and Fig. 15 of Paper 1). There is excellent agreement between the 3 nearly stellar optical peaks $(a, b$, and $c)$ just NW of the quasar nucleus and the three peaks in the radio jet. However, additional apparently aligned structure lies just beyond both radio lobes, with some morphological evidence for association: the optical component $f$ about $3^{\prime \prime}$ beyond the NW radio lobe appears to mimic the latter's structure, and the object $g$ just beyond the SE radio lobe has faint wisps that seem to parallel the radio contours. We have previously discussed some possible interpretations of these two objects and their relation to 3C 212 (Paper 1); here we revisit this discussion to include the results of spectroscopy of these features and of a sample of other faint objects in the field of 3C 212 .

\section{Observations and Data Reduction}

We obtained spectroscopic observations of the 3C212 field with the Low-Resolution Imaging Spectrometer (LRIS) and the Keck I telescope on 1995 October 19 and 20 (UT) and 1996 February 14 and 15 (UT). We used the 600 groove $\mathrm{mm}^{-1}, 7500 \AA$ blaze grating for all observations. The 1995 observations used a $1^{\prime \prime}$ slit and obtained good spectral coverage from $6670 \AA$ to $9300 \AA$. Unresolved

spectral features have FWHM $=4.8 \AA$, and the FWHM of the recorded quasar continuum ranged from $0 . \prime 86$ to $0^{\prime \prime} 98$. The slit was centered on the quasar at PA $-42^{\circ}$; this angle places all of the aligned features on the slit (see Fig. 1 $A$ ). We obtained three $1200 \mathrm{~s}$ integrations on 1995 Oct 19 (UT) through intermittent thin clouds; the first of these was significantly worse than the remaining two (which appear to have suffered negligible extinction) and was subsequently discarded. The following night, we obtained an additional four $1200 \mathrm{~s}$ integrations under photometric conditions. The objects were moved $\sim 5^{\prime \prime}$ along the slit between successive exposures. The 1996 observations were similar, except that most were obtained with 1". 4 -wide slitlets punched in an aperture plate, which also targeted faint galaxies in the field. We obtained twelve $1200 \mathrm{~s}$ integrations with this configuration at a position angle of $-41^{\circ} .4$. We also obtained three $1200 \mathrm{~s}$ integrations with a 0 .'7-wide standard slit at position angle -37.5 , aimed specifically at the bluer portion of the NW component, as well as three $1200 \mathrm{~s}$ integrations in each of two additional aperture plates covering additional galaxies in the field.

The reduction of the CCD frames followed standard procedures, using flat-field frames from 
an internal halogen lamp illuminating the spectrograph cover plate. Airglow lines were dealt with by first using the multiple dithered exposures at each configuration to construct a model of the airglow spectrum, which was then scaled and subtracted from the individual exposures. The long-slit spectra were rectified in the wavelength coordinate from measurements of the spectrum of an internal Ne source. Small corrections to the wavelength zero point were derived from measurements of positions of airglow $\mathrm{OH}$ lines. After this rectification, any remaining residual from the airglow spectrum was removed with the IRAF background task, generally using either a simple median or a linear fit along the columns. We next scanned the spectrum with a series of contiguous 1-pixel-wide apertures covering the region of interest, using tasks in the IRAF apextract package. The scanning parameters were transferred from a trace of the quasar continuum, for which a 6-pixel-wide $\left(1^{\prime \prime} \cdot 3\right)$ aperture was used. This procedure effectively rectifies the spectra in the spatial direction and results in a set of spectra that are aligned with each other in both the spatial and wavelength coordinates. These spectra were calibrated using observations of the spectrophotometric standard star G191B2B (Massey et al. 1988; Massey \& Gronwall 1990). These same observations allowed us to correct for the atmospheric-absorption A and B bands.

The multiaperture spectra were treated similarly. Individual regions corresponding to each slitlet were extracted and reduced independently. The wavelength scale and distortion were determined from $\mathrm{OH}$ airglow lines. Except for the slitlet covering 3C 212 itself and objects $f$ and $g$, we did not attempt to rectify these multiaperture spectra in the spatial coordinate. Instead, we used the IRAF apextract suite of tasks to trace and extract one-dimensional spectra of the objects. The flux calibration is only approximate, having been derived from the longslit observations of the spectrophotometric standards; however, because we kept our targets close to the centerline of the aperture plate, our spectral region is similar to that of the longslit spectra, and the calibration should be fairly accurate.

\section{Results}

\subsection{Spectroscopy of Aligned Components}

Sections of various two-dimensional spectra covering 3C 212 with slits placed along the radio axis are shown in Fig. 俩 $B-E$. There is an emission feature near $7190 \AA$ in the NW aligned component ( $f$ in Fig. 1 $A$ ); this is by far the strongest feature in this object over the observed wavelength range. The feature is clearly a close doublet, and deconvolution into two Gaussian components not only gives the correct wavelength separation for [O II] $\lambda \lambda 3726,3729$ at a redshift of 0.9284 , but it also gives an intensity ratio of 1.5 , which is the expected ratio at low densities. This identification is confirmed by the presence of a much weaker line at $8373 \AA$, the expected position of $\mathrm{H} \gamma$ (Fig. 1 $1 E$ ).

Figure 2 shows traces of the spectra of the NW and SE aligned components beyond the radio lobes $(f$ and $g$ ). In addition to the emission features mentioned above, $f$ shows apparent stellar 
absorption features. Both the strength of the features and the continuum shape over the observed wavelength range are well matched by a single stellar population with an age of 1 Gyr and solar metallicity (Bruzual \& Charlot 1997). However, our HST and groundbased IR images (Paper 1) show that $f$ encompasses a considerable range in color, so a single unreddened stellar population is clearly an oversimplification.

The SE component $g$ also shows stellar absorption features, but no detectible emission lines. The redshift is well determined from the $\mathrm{Ca}$ II H and $\mathrm{K}$ lines to be 1.053, close to the quasar redshift of 1.0489. The continuum color of our spectrum is somewhat redder even than a 10-Gyr-old Bruzual \& Charlot (1997) model (such an age would be unrealistic at this redshift), but on an object this faint, we may be susceptible to small zero-point uncertainties. Taking into account both the absorption features and our (restframe) 0.33-1.0 $\mu \mathrm{m}$ spectral index from Paper 1, we obtain the best overall agreement with a 4-Gyr stellar population model having near-solar metallicity.

\subsection{Spectroscopy of Galaxies in the $3 \mathrm{C} 212$ Field}

The galaxies observed with the aperture plates were selected from a magnitude-limited $(A B[7000 \AA] \leq 25.3)$ sample from a deep image obtained in good seeing with the University of Hawaii 88-inch telescope. The choice of galaxies was controlled partly by our need to obtain deep spectroscopy of the aligned components near 3C 212 itself. The axis of our long-exposure aperture plate was at position angle -41.4 , and we chose galaxies in the available field $\left(7^{\prime}\right.$ in the direction perpendicular to the dispersion) that lay as close as possible to the centerline of the aperture plate, subject to obtaining efficient packing of slitlets, each of which was at least $15^{\prime \prime}$ long. We also tried to place the fainter galaxies on this aperture plate, reserving slightly brighter objects for two additional aperture plates for which we planned shorter total exposures. One of these short-exposure aperture plates was aligned close to the same angle as the long-exposure plate, and the other was at roughly $90^{\circ}$ to it (in both cases, after we had chosen the galaxies, we reoptimized the axes, ending up with position angles of -42.4 and 53.2). While our sample of observed galaxies is neither complete nor unbiased, it should give a reasonable idea of the redshifts of major concentrations of galaxies along the line of sight.

Table 1 shows our results for the 82 different objects (besides the quasar) for which we attempted to obtain spectra. We have firm or probable redshifts for 66 of the objects. Redshifts given in parentheses are mostly objects for which only one emission line is detected, and in most cases the line has been identified as $\mathrm{H} \alpha$. In these cases, we require that the line be both strong and too narrow to be the [O II] $\lambda 3726,3729$ doublet, and that the continuum show no evidence for a discontinuity towards lower levels shortward of the line (so that it is unlikely to be Ly $\alpha$ at high redshift; see related discussion in $\$ 4.3$ ). Figure 3 shows a histogram of the redshift distribution. 


\section{Discussion}

\subsection{The Outer Aligned Components of 3C 212}

We have previously discussed in Paper 1 the problem of the nature of the apparently aligned components $f$ and $g$, which lie beyond the radio lobes of $3 \mathrm{C} 212$, and we will only briefly summarize the main points of that discussion here before considering the new data. The morphological similarity between the optical component $f$ and the NW radio lobe of $3 \mathrm{C} 212$ is truly remarkable (in fact, the initial reaction of some colleagues to whom we showed these data was that we had obviously gotten the scales wrong!). While $g$ shows less obvious structure, it does show faint wisps that appear to extend parallel to the radio contours, and it lies only about $1^{\prime \prime}$ beyond the SW radio hotspot.

The crux of the matter is that, while there appears to be nearly compelling morphological evidence for association rather than chance projection, it is very difficult to understand how any plausible alignment mechanism can produce strong continuum sources beyond the radio lobes. Furthermore, the radial velocity difference of $\gtrsim 18000 \mathrm{~km} \mathrm{~s}^{-1}$ between the quasar and $f$ (particularly coupled with the low internal velocity dispersion of the ionized gas in $f$ ) adds another constraint on possible models for association. The options for $f$ mentioned in Paper 1 were (1) that it was an unassociated foreground object, (2) that its continuum was dominated by optical synchrotron emission associated with undetected radio synchrotron emission (implying a very flat radio - optical spectral index), (3) that its continuum was dominated by inverse Compton scattering of microwave background photons associated with undetected or relic radio radiation (requiring a large total energy in low-energy relativistic electrons), and (4) that the continuum was due to jet-induced star formation marking a previous position of the radio jet or a previous outburst.

For $g$, which is only slightly beyond the SE radio lobe, we considered in Paper 1 the possibility that the faint wisps extending from it could be thermal emission from a bow shock in the ambient medium beyond the radio lobe itself. However, even if this explanation were correct, it cannot explain the bulk of the emission from $g$, for which both the morphology and the extremely red color are inconsistent with those expected from shock-heated gas.

The new observations we have that are relevant to these questions are (1) the deeper spectroscopy of $f$ and $g$, which now show stellar absorption lines in both objects, and (2) the multiaperture spectroscopy of a large number of field galaxies. Our detection of stellar absorption lines in $f$, at the same redshift as the emission lines, effectively eliminates any option in which the continuum is dominated by optical synchrotron or inverse Compton radiation. It also eliminates the possibility that we might have been seeing a combination of emission lines from an intervening object superposed by chance on some form of continuum at or near the quasar redshift. The absorption lines in $g$ finally give us a redshift for this object; the radial velocity difference of $\sim 600$ $\mathrm{km} \mathrm{s}^{-1}$ in the quasar frame strengthens the case for association. 
In the redshift distribution of galaxies in the 3C 212 field (Fig. 3), there is clear concentration near $z \sim 0.925$, with three objects besides $f$ (B32, C23, and C25) falling within about $600 \mathrm{~km} \mathrm{~s}^{-1}$ of this value and one additional object $(\mathrm{C} 05)$ at $z=0.9435$. This clustering in redshift supports the view that $f$ is simply a projected intervening galaxy and that the morphological suggestions of a connection between the radio and optical structure are fortuitious. On the other hand, we have found only two additional galaxies besides $g$ (C17 and $\mathrm{C} 26)$ having redshifts close to that of the quasar; while these galaxies provide evidence for a group around the quasar, there is no evidence for a major cluster.

\subsection{The Redshift Distribution of Galaxies in the $3 \mathrm{C} 212$ Field}

The redshift distribution we observe may be biased by two principal factors: firstly, we selected galaxies with brighter magnitudes for our two shorter-exposure aperture plates than for our long-exposure aperture plate, so we do not have a consistent magnitude limit for the whole sample; secondly, our restricted observed wavelength region and differences in the system efficiency within this region mean that we will be more sensitive to some redshift ranges than to others. Here we estimate the effects of these biases and discuss the likely nature of the galaxies for which we were not able to obtain redshifts. We first take the ratio of the number of galaxies in our observed sample in each one-magnitude interval to that of our total magnitude-limited sample in the same magnitude interval. We can then look at the observed redshift distribution for galaxies in each magnitude interval and make a correction to the total redshift distribution, based on normalizing the distribution of observed galaxies in the magnitude interval to the number in the same interval in the total sample. We then renormalize the resulting distribution back to the total number of our observed sample, rounding to the nearest unit in each bin. Figure $\$ a$ shows the observed and corrected distributions for $\Delta z=0.1$ bins; the two distributions are statistically virtually identical. We now wish to compare our redshift distribution with that found for field galaxies in a large, deep, essentially complete, spectroscopic survey. The published survey best suited for this comparison is that of the $22^{\mathrm{h}}$ field of Cowie et al. (1996; hereinafter "CSHC22 sample"), which is nearly complete to $I=23$. Our survey limit of $A B_{7000}=25.3$ corresponds to $I \approx 24.4$, assuming spectra that are roughly flat in $f_{\lambda}$; but the redshift distribution of our fainter sources is not significantly different from that at $A B_{7000}=24$, so we can use our whole sample in the comparison. Figure $\$ b$ shows the redshift distribution for the CSHC22 sample. This sample comprises 167 galaxies, all but 18 of which have firm redshifts.

The most obvious difference (aside from expected clustering peaks) in the distributions is the presence of galaxies with redshifts nearly up to 2.5 in the CSHC22 sample, whereas no redshifts higher than $\sim 1.4$ are seen in our $3 \mathrm{C} 212$ field redshift distribution. This result is also indicated by a Mann-Whitney $U$ test of the two samples: when the 3C 212 field observed sample is compared

with the whole CSHC22 sample, the probability that the two are drawn from the same population is about $8 \%$, whereas, if we delete galaxies with $z>1.4$ from the latter, the probability rises to 
$>40 \%$. This difference has a natural explanation in terms of the restricted wavelength coverage of our observing configuration. While the wavelength range of our aperture-plate spectra varied somewhat, depending on the exact position of the aperture, both the rapid decline of the CCD response and the increasing strength of the $\mathrm{OH}$ airglow emission work to decrease our detection efficiency at longer wavelengths. In only one case did we identify a line beyond $9200 \AA$ (H $\alpha$ in B31). A galaxy for which [O II] $\lambda 3727$ falls at $9200 \AA$ has $z=1.47$. The next reasonably strong expected emission feature is C III] $\lambda 1909$, which falls below our short-wavelength cutoff for $z \lesssim 2.5$.

This insensitivity to galaxies with redshifts between $\sim 1.47$ and $\sim 2.5$ means that it is quite likely that a significant fraction of the objects for which we were unable to determine redshifts lie within this range. Galaxies with $z>1.4$ constitute $10 \%$ of the CSHC22 sample for which redshifts have been determined; in our sample, we lack redshifts for $20 \%$ of our total, so roughly half (and perhaps more) of these are likely to be high-redshift objects.

\subsection{High-Ionization H II Dwarfs}

One of our faint field galaxies, B08, shows a spectrum dominated by a single strong emission line at $8567 \AA$ on a very weak continuum. The observed equivalent width (EW) of the emission line is $\sim 640 \AA$, and no other features having fluxes greater than $\sim 2 \%$ of this line are detected over an observed wavelength range from 6855 to $9455 \AA$. When a single strong emission line is seen in a low-resolution, wide-bandwidth, optical spectrum of an extragalactic object, it is almost certainly likely to be one of $[\mathrm{O}$ II] $\lambda 3727, \mathrm{Ly} \alpha$, or possibly $\mathrm{H} \alpha$. Other potential identifications either will normally show other nearby lines of reasonable strength or are unlikely to have large equivalent widths. In our spectrum of B08, the line is sufficiently narrow and our resolution sufficiently high that we can eliminate $[\mathrm{O}$ II] $\lambda 3727$ as a possibility: we would have resolved the doublet quite easily.

If the observed line were $\operatorname{Ly} \alpha$, the redshift would be 6.05. However, quite aside from the unprecedented redshift, there is a serious problem with such an identification. Although the observed continuum is very weak, careful measurements in wavelength intervals free of strong airglow emission shows that there is no significant drop in the continuum flux density on the shortward side of the line, as would be expected from Ly $\alpha$ forest absorption. One cannot avoid the problem by supposing that many of the clouds might be essentially wholly ionized at $z \sim 6$, as the flux density $\left(F_{\lambda}\right)$ is still at close to the same level at a wavelength where Ly $\alpha$ has $z=4.6$.

This almost insurmountable difficulty with an identification with Ly $\alpha$ leads us to consider more closely the possibility that the line is $\mathrm{H} \alpha$. While our use of a moderately high dispersion helped us eliminate [O II] as a viable identification, here we are hurt by the fact that our restricted wavelength coverage does not include the expected $\mathrm{H} \beta$ region. The line's large equivalent width (490 $\AA$ in the rest frame, if $\mathrm{H} \alpha$ ) and an upper limit to [N II] of $<1 \%$ of the strength of $\mathrm{H} \alpha$ would seem, at first sight, to argue against this identification. The [S II] $\lambda 6716,6731$ lines would lie in a 
region of strong airglow $\mathrm{OH}$ emission, so the upper limit on their strengths is $\sim 2 \%$ that of the putative $\mathrm{H} \alpha$. Nevertheless, models for starbursts (Leitherer \& Heckman 1995), which have been found to be generally consistent with observations (Stasińska \& Leitherer 1996), indicate that $\mathrm{H} \alpha$ EWs can be as high as 3000 for ages up to $3 \times 10^{6}$ years and can remain above $\sim 300$ up to ages of $10^{8}$ years (these models assume continuous star formation with a Saltpeter luminosity function over a mass range from 0.1 to $\left.100 M_{\odot}\right)$. Furthermore, there are examples of extreme H II galaxies in which metal abundances are low and very hot stars $(>60000 \mathrm{~K})$ are present, suppressing low-ionization species like [N II] and [S II] (e.g., Tol 1214-277; cf. Fig. 4o in Terlevich et al. 1991). Such galaxies tend to show weak emission in He I $\lambda 5876$. This line would fall within our bandpass, and we have a marginal detection, which appears to be confirmed by a careful examination of the two-dimensional spectrum, at about $2 \%$ of the strength of the strong line at $8567 \AA$. We are therefore fairly confident that the latter actually is $\mathrm{H} \alpha$, and this confidence is increased by the presence of another object, D12, in our sample, which also has a very weak continuum and strong lines; it would have caused similar problems of identification had it not had a sufficiently high redshift that the [O III] lines fall within our bandpass. Because such dwarfs dominated by very young, metal-poor populations and showing high ionizations appear not to be too uncommon, it is clear that seeing a single, strong line with a large equivalent width is not sufficient for an identification with Ly $\alpha$. Fortunately, most spectroscopic surveys of faint galaxies have wider wavelength coverage than we had and will normally observe the expected [O III] region for any lines that might be $\mathrm{H} \alpha$ candidates. The strongest evidence in favor of $\mathrm{Ly} \alpha$ for lines in this region of the spectrum (i.e., redward of $\sim 6600 \AA$ ) would be a clear indication of a continuum discontinuity across the emission line.

\section{Summary}

We have obtained deep spectroscopy of the two objects $f$ and $g$, which lie respectively just beyond the NW and SE radio lobes of 3C 212. Object $f$ shows both emission lines and absorption lines from a moderately-young stellar population at a redshift of 0.9284 , blueshifted

by some $18,000 \mathrm{~km} \mathrm{~s}^{-1}$ relative to the quasar. Object $g$ has the spectrum of an old ( $Z 4$ Gyr) stellar population at a redshift close to that of the quasar and is likely associated with it. Our multiaperture spectroscopy of 82 objects in the 3C 212 field has turned up 2 additional galaxies with redshifts close to that of $3 \mathrm{C} 212$, both at fairly large angular separations from the quasar, as well as 3 galaxies having redshifts close to that of $f$. At both of these redshifts, the galaxies all lie within a projected distance of about $1 \mathrm{Mpc}$ of $3 \mathrm{C} 212$ and of $f\left(H_{0}=75 \mathrm{~km} \mathrm{~s}^{-1} \mathrm{Mpc}^{-1}, q_{0}=1 / 2\right)$. Thus, it appears that both 3C 212 and $f$ are members of rather loose groups; more importantly, in spite its morphological similarity to the NW radio lobe of 3C 212 and its precise alignment with the radio jet, $f$ is probably an unrelated foreground object.

The redshift distribution of faint galaxies in the 3C 212 field agrees well with that of the Cowie et al. (1996) $22^{\mathrm{h}}$ field except for a lack of galaxies with $z \gtrsim 1.4$ in the former. This difference 
is readily understood in terms of our lack of coverage of wavelength regions in which any strong emission lines will fall for $1.47 \lesssim z \lesssim 2.5$, and it suggests that a large fraction of our objects without redshifts lie within this range.

The presence of two young dwarf starburst galaxies with high ionizations among the fainter members of our sample indicates that such objects are likely to be fairly common in field-galaxy samples at $R \gtrsim 24$.

We thank Len Cowie and Esther Hu for discussions and for providing a machine-readable version of Table 1 from Cowie et al. (1996). We have enjoyed discussions of the alignment effect in 3C 212 with many people, including Neil Trentham, Mark Lacy, and Ken Chambers. We thank Zachery Ortogero for helping with some of the figures. This research was partially supported by NASA through Grant No. GO-05401.01-93A from the Space Telescope Science Institute, which is operated by AURA, Inc., under NASA Contract No. NAS 5-26555, and by NSF under grant AST95-29078. SER was supported by a PPARC Postdoctoral Fellowship.

\section{REFERENCES}

Bruzual, G., \& Charlot, S. 1997, in preparation

Chambers, K.C., Miley, G., \& van Breugel, W. 1987, Nature, 329, 609

Cowie, L.L., Songaila, A., Hu, E.M., \& Cohen, J.G. 1996, AJ, 112, 839

Dickinson, M., Dey, A., \& Spinrad, H. 1995, in "Galaxies in the Young Universe", ed. H. Hippelein, K. Meisenheimer, \& Röser, H.-J., Springer-Verlag, p. 164

di Serego Alighieri, S., Cimatti, A., \& Fosbury, R.A.E. 1994, ApJ, 431, 123

Leitherer, C., \& Heckman, T.M. 1995, ApJS, 96, 9

Longair, M.S., Best, P.N., \& Röttgering, H.J.A. 1995, MNRAS, 275, L47.

Massey, P., Strobel, K., Barnes, J.V., \& Anderson, E. 1988, ApJ, 328, 315.

Massey, P. \& Gronwall, C. 1990, ApJ, 358, 344.

McCarthy, P.J., van Breugel, W., Spinrad, H., \& Djorgovski, S. 1987, ApJ, 321, L29

Ridgway, S.E, \& Stockton, A. 1997, AJ, 114, 511 (Paper 1)

Stanińska, G. \& Leitherer, C. 1996, ApJS, 107, 661

Stockton, A., Ridgway, S.E., \& Kellogg, M. 1996, AJ, 112, 902

Terlevich, R., Melnick, J., Masegosa, J., Moles, M., \& Copetti, M.V.F. 1991 A\&AS, 91, 285 
Table 1. Spectroscopy of 3C 212 Field Objects

\begin{tabular}{|c|c|c|c|c|c|}
\hline Object & $\Delta \alpha^{\mathrm{a}}$ & $\Delta \delta^{\mathrm{a}}$ & $A B_{7000} \mathrm{~b}$ & $z$ & Notes $^{\mathrm{c}}$ \\
\hline B02 & $-109 . .5$ & $105{ }^{\prime \prime} 9$ & 24.8 & 1.311 & [O II], Ca II, CN \\
\hline B03 & $-88^{\prime \prime} .7$ & $108^{\prime \prime} \cdot 2$ & 24.5 & 0.6671 & $\mathrm{H} \beta,[\mathrm{O} \mathrm{III}]$ \\
\hline B04 & $-94^{\prime \prime} 8$ & $83^{\prime \prime} 3$ & 25.2 & $\cdots$ & $\mathrm{FBC}$ \\
\hline B05 & $-75 !^{\prime \prime} 0$ & $79^{\prime \prime} 8$ & 24.6 & 1.1386 & {$[\mathrm{O} \mathrm{II}],[\mathrm{Ne} \mathrm{III}]$} \\
\hline B06 & $-63 !^{\prime \prime} 8$ & $68^{\prime \prime} 9$ & 24.6 & $\cdots$ & NDC \\
\hline B07 & $-64^{\prime \prime} 7$ & $54^{\prime \prime} 0$ & 24.2 & $\cdots$ & $\mathrm{FBC}$ \\
\hline B08 & $-40^{\prime \prime} 9$ & $57 !^{\prime \prime} 8$ & 24.9 & 0.3053 & $\mathrm{H} \alpha, \mathrm{He} \mathrm{I} \lambda 5876$ \\
\hline B09 & $-30^{\prime \prime} 5$ & $49{ }^{\prime \prime} 6$ & 24.9 & 1.402 & {$[\mathrm{O} \mathrm{II}],[\mathrm{Ne} \mathrm{III}]$} \\
\hline $\mathrm{B} 10$ & $-30^{\prime \prime} 1$ & $35^{\prime \prime} 2$ & 24.8 & 0.6052 & $\mathrm{H} \beta,[\mathrm{O} \mathrm{III}]$ \\
\hline B11 & $-13^{\prime \prime} 5$ & $29 .^{\prime \prime} 3$ & 23.7 & $\cdots$ & em. line at $9170.1 \AA$ \\
\hline $\mathrm{B} 12$ & $-7 !^{\prime \prime} 6$ & $18^{\prime \prime} 9$ & 23.6 & 0.3380 & $\mathrm{H} \alpha,[\mathrm{N} \mathrm{II}]$ \\
\hline B13 & $-5{ }^{\prime \prime} 2$ & $6^{\prime \prime} 3$ & 22.6 & 0.9284 & $f ;[\mathrm{OII}], \mathrm{H} \gamma($ see text $)$ \\
\hline B14 & $0 .{ }^{\prime \prime} 0$ & $0^{\prime \prime} 0$ & 19.0 & 1.0489 & $3 \mathrm{C} 212$ \\
\hline B15 & $3 . \prime 9$ & $-4^{\prime \prime} 2$ & 25.3 & 1.053 & $g$; Ca II (see text) \\
\hline $\mathrm{B} 16$ & $33^{\prime \prime} 3$ & $2 . .3$ & 24.6 & 1.293 & $\mathrm{CaII}$ \\
\hline B17 & $1^{\prime \prime} 3$ & $-35^{\prime \prime} 3$ & 24.1 & $\cdots$ & $\mathrm{FBC}$ \\
\hline B18 & $57 !^{\prime \prime} 4$ & $-8 .^{\prime \prime} 4$ & 24.8 & $\cdots$ & NDC \\
\hline B19 & $566^{\prime \prime} 4$ & $-37 !^{\prime \prime} 0$ & 23.8 & $\cdots$ & Continuum contaminated by star \\
\hline $\mathrm{B} 20$ & $75 !^{\prime \prime} 5$ & $-37 ! ! 8$ & 24.9 & 0.4881 & $\mathrm{H} \beta,[\mathrm{O} \mathrm{III}]$ \\
\hline $\mathrm{B} 21$ & $89 !^{\prime \prime} 0$ & $-41{ }^{\prime \prime} 8$ & 24.9 & $(0.1347)$ & $\mathrm{H} \alpha$ \\
\hline $\mathrm{B} 22$ & $78 !^{\prime \prime} 0$ & $-677^{\prime \prime} 5$ & 25.3 & 0.4880 & {$[\mathrm{O} \mathrm{III}]$} \\
\hline B23 & $77^{\prime \prime} 7$ & $-82^{\prime \prime} 7$ & 24.5 & 0.7144 & $\mathrm{H} \beta,[\mathrm{O} \mathrm{III}]$ \\
\hline B24 & $93{ }^{\prime \prime} 7$ & $-89 !^{\prime \prime} 2$ & 23.8 & 1.195 & [O II], Balmer abs. \\
\hline $\mathrm{B} 25$ & $86^{\prime \prime} 5$ & $-115^{\prime \prime} 0$ & 24.8 & 0.4307 & $\mathrm{H} \beta,[\mathrm{O} \mathrm{III}]$ \\
\hline B26 & $110 . \prime 4$ & $-102^{\prime \prime} \cdot 2$ & 23.4 & 0.6842 & $\mathrm{H} \beta,[\mathrm{O} \mathrm{III}], \mathrm{H} \gamma$ \\
\hline $\mathrm{B} 27$ & $112^{\prime \prime} 0$ & $-104^{\prime \prime} 3$ & 24.4 & 0.6831 & $\mathrm{H} \beta, \mathrm{H} \gamma$ \\
\hline $\mathrm{B} 28$ & $114 !^{\prime \prime} 6$ & $-107 ! ! 5$ & 23.3 & 0.6841 & $\mathrm{H} \beta,[\mathrm{O} \mathrm{III}], \mathrm{H} \gamma$ \\
\hline B29 & $1322^{\prime \prime} 1$ & $-109 .{ }^{\prime \prime} 1$ & 24.2 & 0.7569 & $\mathrm{H} \beta,[\mathrm{O} \mathrm{III}]$ \\
\hline B30 & $113^{\prime \prime} 6$ & $-144^{\prime \prime} 2$ & 23.7 & 0.2995 & $\mathrm{H} \alpha,[\mathrm{S} \mathrm{II}]$ \\
\hline B31 & $148^{\prime \prime} 5$ & $-137^{\prime \prime} 6$ & 24.3 & 0.442 & $\mathrm{H} \alpha, \mathrm{H} \beta$ \\
\hline B32 & $120^{\prime \prime} 7$ & $-180^{\prime \prime} 4$ & 23.1 & 0.9290 & {$[\mathrm{O} \mathrm{II}]$} \\
\hline B33 & $138 !^{\prime \prime} 8$ & $-187^{\prime \prime} 8$ & 24.9 & $\cdots$ & $\mathrm{FBC}$ \\
\hline B34 & $134^{\prime \prime} 7$ & $-203^{\prime \prime} 1$ & 24.8 & 0.6434 & $\mathrm{H} \beta,[\mathrm{O} \mathrm{III}]$ \\
\hline B35 & $185 . \prime 6$ & $-179^{\prime \prime} 0$ & 23.8 & $(0.2267)$ & $\mathrm{H} \alpha$ \\
\hline $\mathrm{C} 02$ & $106^{\prime \prime} 9$ & $98^{\prime \prime} 6$ & 23.6 & $\cdots$ & $\mathrm{FBC}$ \\
\hline $\mathrm{C} 03$ & $111^{\prime \prime} 1$ & $63^{\prime \prime} 2$ & 22.9 & $\cdots$ & $\mathrm{FBC}$ \\
\hline $\mathrm{C} 04$ & $103^{\prime \prime} 7$ & $57 !^{\prime \prime} 4$ & 23.9 & $(0.3150)$ & $\mathrm{H} \alpha$, poss. $[\mathrm{N} \mathrm{III}]$ \\
\hline $\mathrm{C} 05$ & $52^{\prime \prime} 9$ & $87^{\prime \prime} 7$ & 22.9 & 0.9435 & {$[\mathrm{O} \mathrm{II}],[\mathrm{Ne} \mathrm{III}]$} \\
\hline $\mathrm{C} 06$ & $74^{\prime \prime} 0$ & $35 !^{\prime \prime} 8$ & 23.8 & 0.7614 & $\mathrm{H} \beta,[\mathrm{O} \mathrm{III}]$ \\
\hline $\mathrm{C} 07$ & $44^{\prime \prime} 1$ & $36^{\prime \prime} 4$ & 22.3 & 0.3136 & $\mathrm{H} \alpha,[\mathrm{N} \mathrm{II}]$ \\
\hline $\mathrm{C} 08$ & $33 !^{\prime \prime} 9$ & $27 !^{\prime \prime} 9$ & 22.8 & 0.3323 & $\mathrm{H} \alpha,[\mathrm{N} \mathrm{II}]$ \\
\hline $\mathrm{C} 09$ & $31^{\prime \prime} 6$ & $13^{\prime \prime} 9$ & 22.9 & 0.7032 & $\mathrm{H} \beta,[\mathrm{O}$ III $]$ \\
\hline $\mathrm{C} 10$ & $21^{\prime \prime} 1$ & $3 ! \prime 3$ & 23.1 & 0.4921 & $\mathrm{H} \beta,[\mathrm{O} \mathrm{III}]$ \\
\hline $\mathrm{C} 11$ & $-5^{\prime \prime} 2$ & $6^{\prime \prime} 3$ & 22.6 & 0.9283 & $f$ (same as B13); [O II $]$ \\
\hline $\mathrm{C} 12$ & $-13^{\prime \prime} 8$ & $-4^{\prime \prime} 5$ & 23.0 & $\cdots$ & FBC \\
\hline $\mathrm{C} 13$ & $-32^{\prime \prime} 9$ & $-14^{\prime \prime} 5$ & 23.0 & 0.5167 & {$[\mathrm{O}$ III $]$} \\
\hline $\mathrm{C} 14$ & $-53{ }^{\prime \prime} 4$ & $-21^{\prime \prime} 9$ & 22.0 & 0.4884 & $\mathrm{H} \beta,[\mathrm{O} \mathrm{III}]$ \\
\hline $\mathrm{C} 15$ & $-61{ }^{\prime \prime} 8$ & $-34^{\prime \prime} 3$ & 23.3 & 0.4201 & [O III $]$ \\
\hline $\mathrm{C} 16$ & $-56^{\prime \prime} 3$ & $-58^{\prime \prime} 5$ & 23.0 & $\ldots$ & $\mathrm{FBC}$ \\
\hline $\mathrm{C} 17$ & $-80^{\prime \prime} 6$ & $-54^{\prime \prime} 1$ & 22.9 & 1.0505 & CaII, G-band \\
\hline $\mathrm{C} 18$ & $-79 .^{\prime \prime} 7$ & $-79^{\prime \prime} 9$ & 21.2 & 0.3056 & $\mathrm{H} \alpha,[\mathrm{N} \mathrm{II}],[\mathrm{S} \mathrm{II}]$ \\
\hline C19 & $-114^{\prime \prime} 5$ & $-52{ }^{\prime \prime} 8$ & 22.2 & 0.5100 & $\mathrm{H} \beta,[\mathrm{O} \mathrm{III}]$ \\
\hline
\end{tabular}


Table 1 - Continued

\begin{tabular}{|c|c|c|c|c|c|}
\hline Object & $\Delta \alpha^{\mathrm{a}}$ & $\Delta \delta^{\mathrm{a}}$ & $A B_{7000} \mathrm{~b}$ & $z$ & Notes $^{\mathrm{c}}$ \\
\hline $\mathrm{C} 20$ & $-114^{\prime \prime} 3$ & $-86 .{ }^{\prime \prime} 7$ & 22.6 & 0.8070 & Ca II \\
\hline $\mathrm{C} 21$ & $-128^{\prime \prime} 1$ & $-89 . \prime 5$ & 23.7 & 1.397 & {$[\mathrm{O} \mathrm{II}]$} \\
\hline $\mathrm{C} 22$ & $-1355^{\prime \prime} 0$ & $-121^{\prime \prime} 0$ & 22.7 & 0.8318 & $\mathrm{H} \beta,[\mathrm{O} \mathrm{III}]$ \\
\hline $\mathrm{C} 23$ & $-141^{\prime \prime} 4$ & $-125^{\prime \prime} 5$ & 22.1 & 0.9221 & {$[\mathrm{O} \mathrm{II}]$} \\
\hline $\mathrm{C} 24$ & $-156^{\prime \prime} 6$ & $-1222^{\prime \prime} 4$ & 22.5 & 0.7575 & $\mathrm{H} \beta,[\mathrm{O} \mathrm{III}]$ \\
\hline $\mathrm{C} 25$ & $-168{ }^{\prime \prime} 3$ & $-123 .^{\prime \prime} 8$ & 21.4 & $0.924:$ & $4000 \AA$ break \\
\hline $\mathrm{C} 26$ & $-175^{\prime \prime} 1$ & $-154^{\prime \prime} \cdot 2$ & 22.3 & 1.043 & {$[\mathrm{O} I \mathrm{II}],[\mathrm{Ne} \mathrm{III}]$, poss. $\mathrm{H} \gamma$} \\
\hline $\mathrm{C} 27$ & $-202^{\prime \prime} 9$ & $-144^{\prime \prime} 9$ & 22.6 & 0.7108 & $\mathrm{H} \beta,[\mathrm{O} \mathrm{III}]$ \\
\hline D01 & $-123^{\prime \prime} .5$ & $88^{\prime \prime} 4$ & 23.2 & $(0.1790)$ & $\mathrm{H} \alpha$ \\
\hline D02 & $-65^{\prime \prime} 4$ & $111^{\prime \prime} 6$ & 22.8 & $\ldots$ & $\mathrm{FBC}$ \\
\hline D03 & $-78^{\prime \prime} 2$ & $77 !^{\prime \prime} 1$ & 22.9 & $(0.6256)$ & $\mathrm{H} \beta$, poss. [O III], $\mathrm{H} \gamma$ \\
\hline D04 & $-74^{\prime \prime} 8$ & $60{ }^{\prime \prime} 3$ & 23.5 & $\ldots$ & $\mathrm{FBC}$ \\
\hline D05 & $-64^{\prime \prime} 7$ & $41^{\prime \prime} 5$ & 20.3 & 0.3294 & $\mathrm{H} \alpha,[\mathrm{N} \mathrm{II}], \mathrm{Na} \mathrm{D}$ \\
\hline D06 & $-72^{\prime \prime} 9$ & $11^{\prime \prime} 9$ & 22.7 & 0.000 & M4 dwarf star \\
\hline D07 & $-33^{\prime \prime} \cdot 7$ & $26^{\prime \prime} 3$ & 23.0 & 0.3051 & $\mathrm{H} \alpha,[\mathrm{N} \mathrm{II}]$ \\
\hline D08 & $-4^{\prime \prime} 4$ & $37 !^{\prime \prime} 4$ & 21.2 & 0.3301 & $\mathrm{H} \alpha,[\mathrm{N} \mathrm{II}]$ \\
\hline D09 & $-28^{\prime \prime} 1$ & $-4^{\prime \prime} 1$ & 21.1 & 0.3331 & $\mathrm{H} \alpha,[\mathrm{S} \mathrm{II}]$ \\
\hline D10 & $-15^{\prime \prime} 1$ & $-12 !^{\prime \prime} 5$ & 23.8 & $(0.136)$ & $\mathrm{H} \alpha$ \\
\hline D11 & $-11^{\prime \prime} 2$ & $-16^{\prime \prime} 8$ & 22.5 & 0.8091 & $\mathrm{H} \epsilon$ and higher Balmer abs. \\
\hline D12 & $11^{\prime \prime} 1$ & $-21{ }^{\prime \prime} 3$ & 23.8 & 0.4139 & {$[\mathrm{O} \mathrm{III}], \mathrm{H} \alpha$} \\
\hline D13 & $50^{\prime \prime} 2$ & $-22^{\prime \prime} 4$ & 22.0 & 0.5365 & $\mathrm{H} \beta,[\mathrm{O} \mathrm{III}]$ \\
\hline D14 & $71^{\prime \prime} 6$ & $-39^{\prime \prime} 5$ & 22.4 & 0.5086 & $\mathrm{H} \beta,[\mathrm{O} \mathrm{III}]$ \\
\hline D15 & $69 . \prime 1$ & $-73^{\prime \prime} 0$ & 22.6 & 0.1140 & $\mathrm{H} \alpha,[\mathrm{S} \mathrm{II}]$ \\
\hline D16 & $100 !^{\prime \prime} 8$ & $-69^{\prime \prime} 5$ & 22.7 & 0.7147 & $\mathrm{H} \beta,[\mathrm{O} \mathrm{III}]$ \\
\hline D17 & $86^{\prime \prime} 4$ & $-98^{\prime \prime} 6$ & 23.8 & $(0.3179)$ & $\mathrm{H} \alpha$ \\
\hline D18 & $90^{\prime \prime} 3$ & $-111^{\prime \prime} 5$ & 21.6 & 0.6834 & $\mathrm{H} \beta, \mathrm{H} \gamma$ \\
\hline D19 & $114^{\prime \prime} 0$ & $-113^{\prime \prime} 6$ & 22.7 & 0.6837 & $\mathrm{H} \beta, \mathrm{H} \gamma$ \\
\hline D20 & $999^{\prime \prime} 9$ & $-142^{\prime \prime}{ }^{3}$ & 23.4 & $\ldots$ & $\mathrm{FBC}$ \\
\hline D21 & $105^{\prime \prime} 4$ & $-150 .{ }^{\prime \prime} 5$ & 23.1 & $\cdots$ & poss. em. line at $8302.4 \AA$ \\
\hline D22 & $143^{\prime \prime} 3$ & $-137^{\prime \prime} 0$ & 22.8 & 0.3560 & $\mathrm{H} \alpha,[\mathrm{N} \mathrm{II}]$ \\
\hline D23 & $169 \prime^{\prime \prime} 3$ & $-142^{\prime \prime} 5$ & 22.6 & $(0.6439)$ & {$[\mathrm{O}$ III $]$} \\
\hline D24 & $1333^{\prime \prime} 8$ & $-188^{\prime \prime} 9$ & 23.4 & 0.6819 & $\mathrm{H} \beta,[\mathrm{O} \mathrm{III}], \mathrm{H} \gamma$ \\
\hline D25 & $1400^{\prime} 1$ & $-195^{\prime \prime} 1$ & 23.0 & 0.7545 & $\mathrm{H} \beta,[\mathrm{O} \mathrm{III}]$ \\
\hline
\end{tabular}

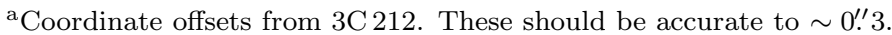

${ }^{\mathrm{b}} A B$ magnitude in a $4^{\prime \prime}$ diameter aperture, from a bandpass centered at $7000 \AA$ and having a FWHM of $2000 \AA$. A $1-\sigma$ detection above sky would correspond to $A B_{7000}=25.8$.

${ }^{\mathrm{c}}$ Includes spectral features on which the redshift is based; however, not all of these features have necessarily been used to determine the value of the redshift. Emission features: [O II] $\lambda \lambda 3726,3729,[\mathrm{Ne}$ III] $\lambda 3868, \mathrm{H} \gamma \lambda 4340$, He I $\lambda 5876, \mathrm{H} \beta \lambda 4861,[\mathrm{O} \mathrm{III]} \lambda \lambda 4959,5007, \mathrm{H} \alpha \lambda 6563$, [N II] $\lambda 6583$, [S II] $\lambda \lambda 6716,6731$. Absorption features: $\mathrm{H} \epsilon$ and higher Balmer lines, Ca II $\mathrm{H}$ and $\mathrm{K}$, NaI D. Other abbreviations: $\mathrm{FBC}=$ faint blue continuum; NDC $=$ no detected continuum.

Note. - Redshifts in parentheses depend on only one line (usually identified as $\mathrm{H} \alpha$ ) or are otherwise uncertain. 
Fig. 1. - HST image and spectra of objects near 3C 212. (A) HST image, aligned so that position angle -41.4 is vertical. Object designations are the same as in Ridgway \& Stockton (1997). The 3.6-cm VLA map is overlain as green contours. Tickmarks are at $1^{\prime \prime}$ intervals. ( $\left.B-D\right)$ Keck LRIS spectral maps of the region of the [O II] $\lambda 3727$ doublet in object $f$. The dispersion direction is horizontal, and the vertical spatial coordinate is aligned with that of image $(A)$. (B) Combination of 4 hours of data with a 1."4 slit at PA -41.4 and 2 hours of data with a $1^{\prime \prime}$ slit at PA $-42^{\circ} ;(C)$ 2 hours with a $1^{\prime \prime}$ slit at PA $-42^{\circ}$ alone; (D) 1 hour with a $0^{\prime \prime} 7$ slit at PA $-37^{\circ} .5$. (E) From the same combined spectrum as $(B)$, but showing the $\mathrm{H} \gamma$ line. 


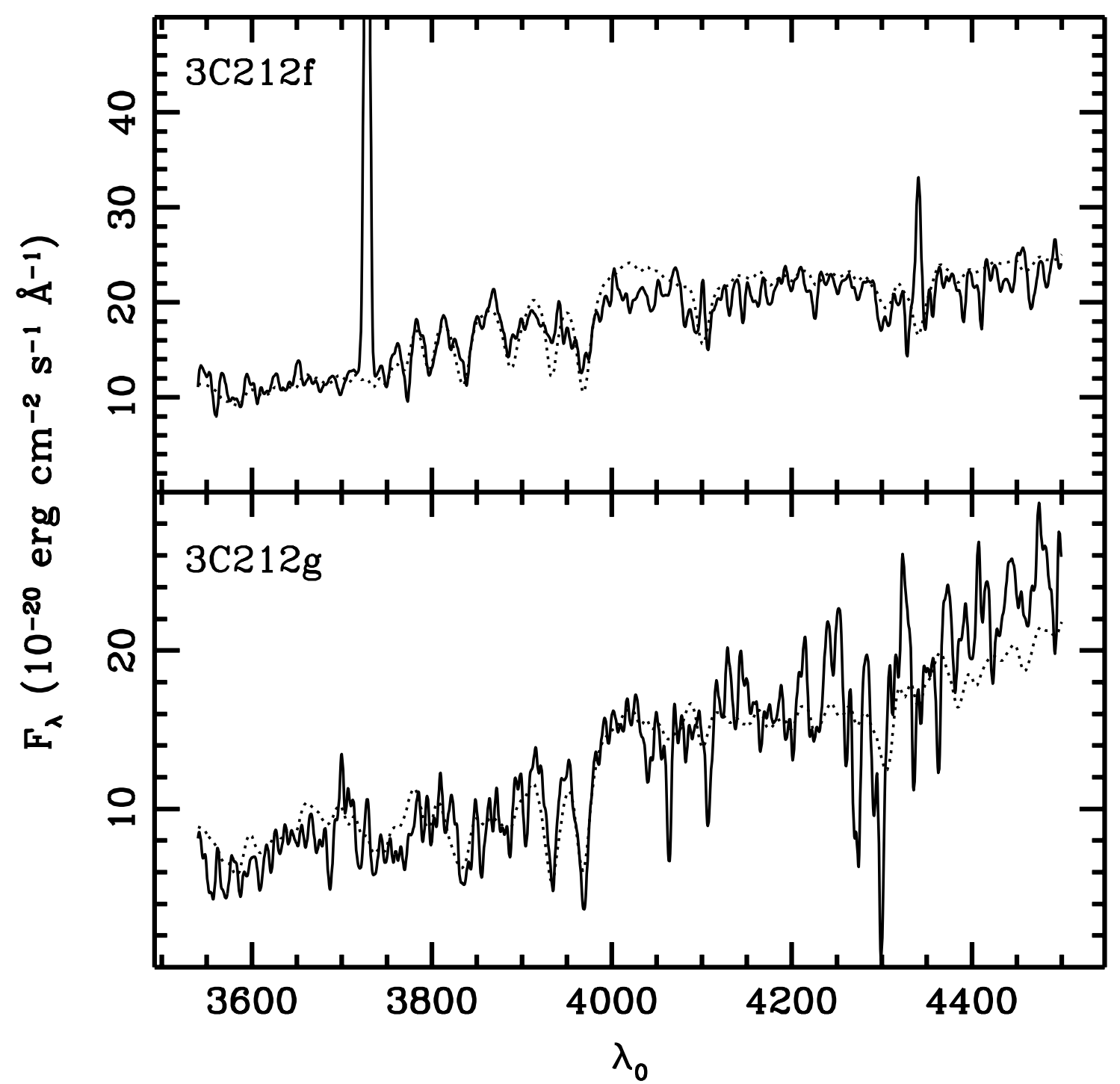

Fig. 2.- Plots of spectra of $f$ and $g$, from the 6 -hour combined spectrum obtained with $1^{\prime \prime}$ and $1^{\prime \prime} 4$ slits. Both spectra have been restored to the rest frame, using $z=0.9283$ for $f$ and $z=1.053$ for $g$. The dotted traces superposed on the data are of single-epoch solar-metallicity Bruzual-Charlot (1995) isochronal synthesis models: that for $f$ has an age of $1.0 \mathrm{Gyr}$, and that for $g$ has an age of 4 Gyr. 


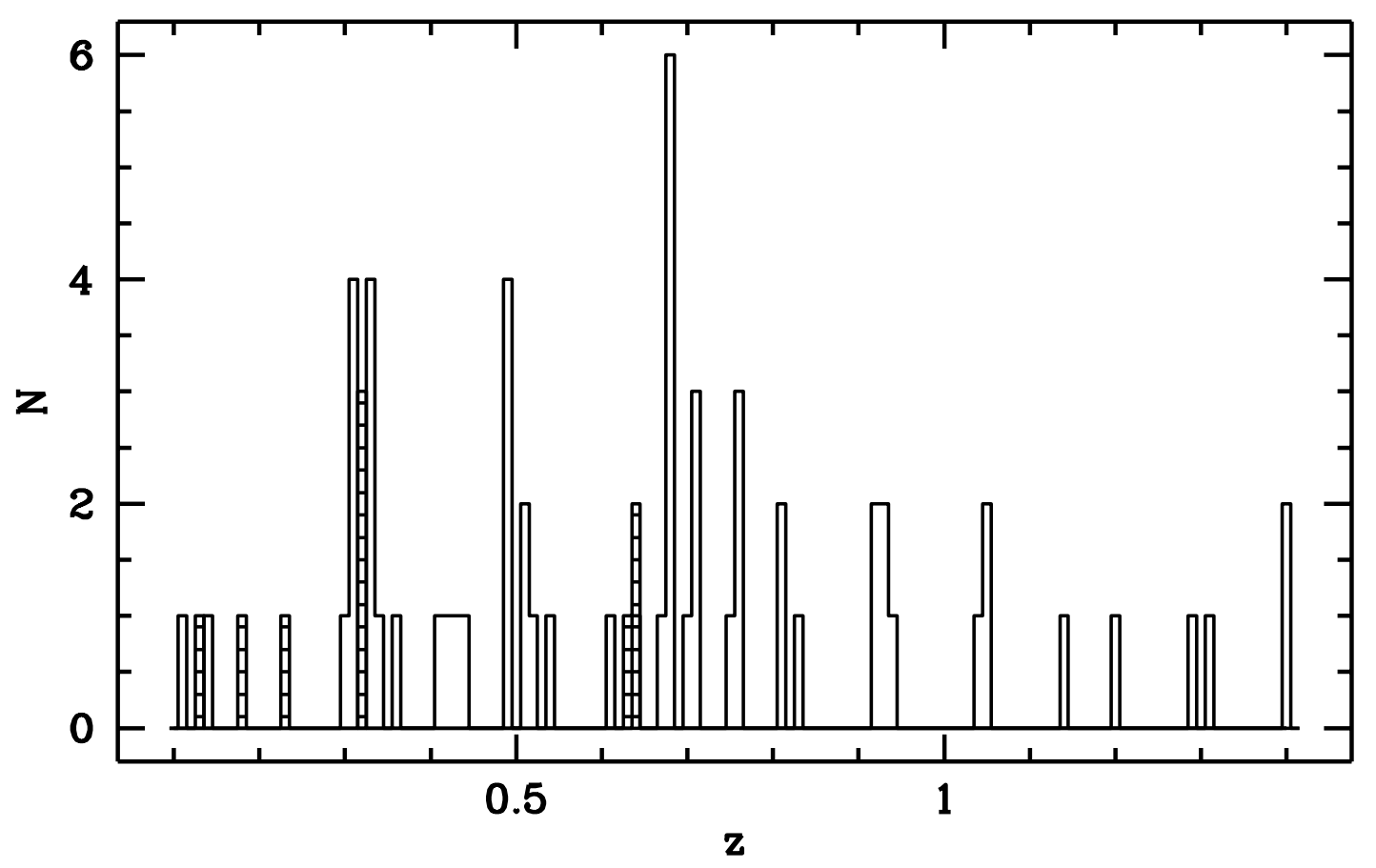

Fig. 3.- Histogram of the redshift distribution of galaxies observed in the $3 \mathrm{C} 212$ field. 


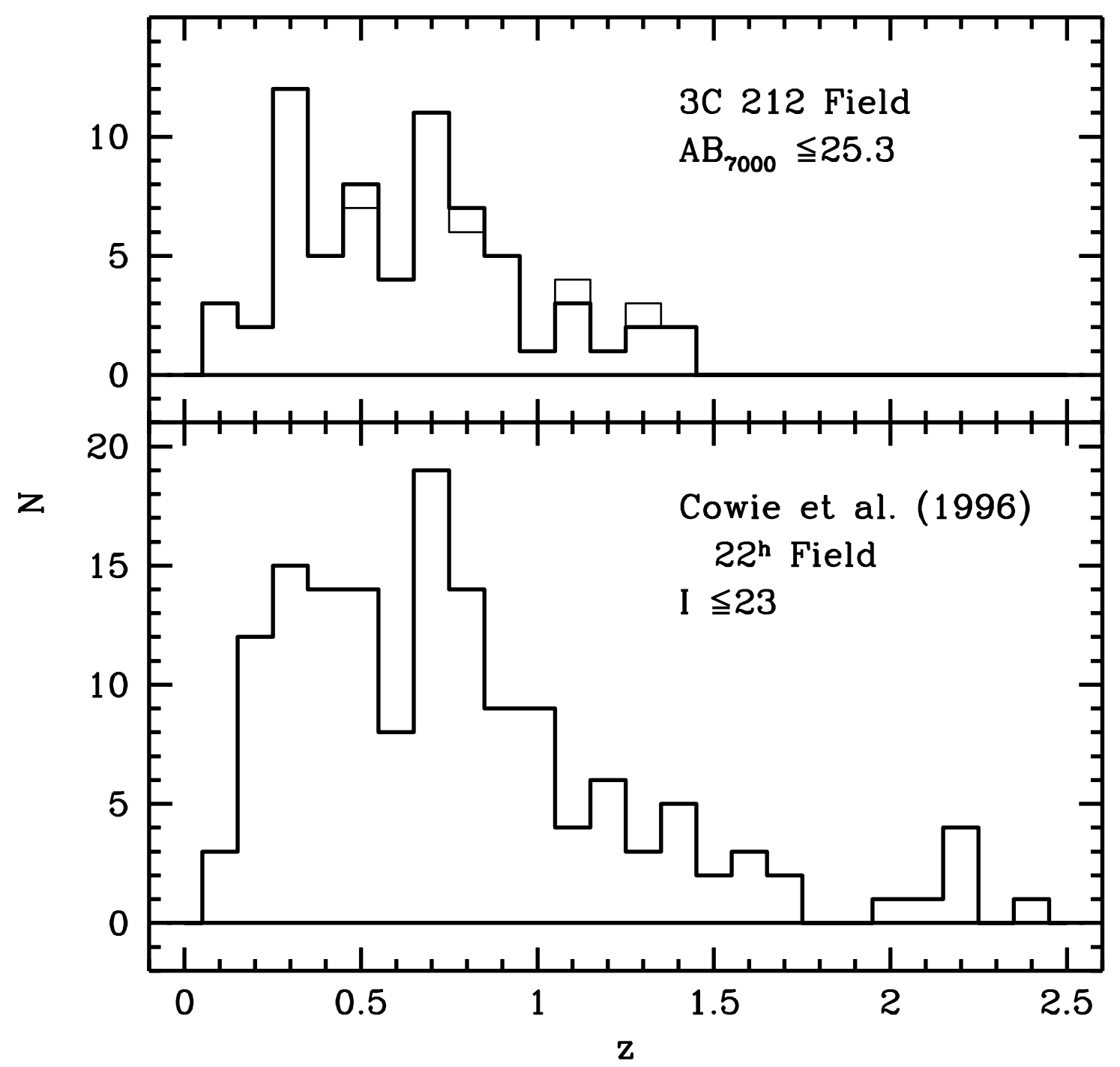

Fig. 4.- Comparison of redshift distributions. (a) heavy line - the same redshift distribution of the 3C 212 field as shown in Fig. 3, binned to 0.1 intervals in z. lighter line - redshift distribution after a statistical correction for selection bias in the sample, as described in the text. (b) Distribution of redshifts for the $22^{\mathrm{h}}$ field of Cowie et al. (1996). 
This figure "fig1.jpg" is available in "jpg" format from: http://arxiv.org/ps/astro-ph/9801056v1 